\title{
Morbi-RSA - Das Ende der DMP?
}

Die aktuellen wissenschaftlichen Auswertungen von DMP erlauben keine validen Aussagen über deren Nutzung und Wirtschaftlichkeit. Mit der Einführung des Morbi-RSA werden die finanziellen Anreize für eine flächendeckende Patientenrekrutierung entfallen. Nur kosteneffektive DMP werden dann noch eine Überlebenschance haben.

\section{Christoph Straub, Eva Susanne Dietrich}

Hauptziel der Disease Management Programme (DMP) war die Verbesserung des Behandlungsablaufs und der Qualität der medizinischen Versorgung chronisch Kranker. Dazu sollten DMP eine abgestimmte, koordinierte Versorgung über Sektorengrenzen hinweg auf der Basis der besten verfügbaren wissenschaftlichen Evidenz beinhalten (1).

Wurde dieses Ziel erreicht? Hat sich der enorme Aufwand bei Krankenkassen und Ärzten gelohnt?

In der Regel wurden DMP-Verträge landesweit von allen Kassen gemeinsam - oder zwar einzeln aber zu den gleichen Konditionen - mit den Kassenärztlichen Vereinigungen abgeschlossen. Die Leitlinien der Fachgesellschaften sind nicht zwangsläufig deckungsgleich mit den Therapiekorridoren, die in den DMP festgelegt sind und die sich stark an sozioökonomischen Aspekten orientieren. Der Bezug auf die aktuell beste Evidenz blieb in der Vergangenheit mehr als einmal auf der Strecke (2).

Die Monopolposition der KVen wurde weiter gestärkt, anstelle auch hier den Qualitätswettbewerb zu intensivieren. Die geplante Einbindung von Krankenhäusern und Reha-Trägern in die Programme hat sich beim DMP Diabetes zwar in der letzten Zeit deutlich verbessert, ist aber immer noch unzureichend.

Derzeit profitiert die Kasse, die möglichst viele Versicherte in die DMP-Programme aufnimmt. Die in der Verordnung über das Verfahrenzum Risikostrukturausgleich in der GKV ((RSAV) vorgegebene Grundbedingung, dass nur Versicherte in die Programme eingeschrieben werden dürfen, bei denen durch die intensivierte Betreuung eine Verbesserung der Lebensqualität zu erwarten ist, wird nicht überprüft (3).

Die größten Optimierungspotentiale existieren bei Patienten, die bisher nicht behandelt wurden. Bei DiabetesII-Patienten sind dies nach Auswertungen der TK zwischen 11 und 25 Prozent.

Außerdem wird nur ein Teil der Typ-II-Diabetiker mit hohem Blutdruck und hohen Bluttfettwerten diesbezüg-

Christoph Straub, TK, Hamburg; Eva Susanne Dietrich, Wissenschaftliches Institut der TK für Nutzen und Effizienz im Gesundheitswesen (WINEG), Hamburg lich behandelt, obwohl hier ein Potential für eine Verhinderung von teuren Folgekomplikationen wie Myokardinfarkt oder Schlaganfällen besteht (4).

Die Reduktion des Risikos, eine Komplikation zu erleiden, ist zudem maßgeblich von verhaltensbedingten Faktoren wie Ernährung, Bewegung und Rauchen abhängig. Nur ein Achtel aller an Diabetes-II-Erkrankten ist in der Lage, durch die Veränderung des Verhaltens langfristig die erforderlichen Zielwerte einzuhalten (4).

Können DMP in der Praxis die Versorgungsqualität verbessern, auch wenn ein breiter, unfokussierter Patienteneinschluss erfolgt? Die Antwort auf diese Frage muss offen bleiben. Denn es gibt bisher keinen wissenschaftlichen Beleg, dass die DMP wirksam sind, obwohl eine Reihe von aufwändigen Studien in den letzten Jahren durchgeführt wurde.

Sicher gibt es eine Reihe von Hinweisen auf mögliche Effekte. So zeigte eine Studie von Infas Bonn, Prognos Düsseldorf und WIAD bei 1,25 Millionen AOK-Versicherten, die an DMP zu Diabetes mellitus Typ II teilnahmen, dass der Blutdruck der Hypertonie-Patienten innerhalb von drei Jahren im Durchschnitt von diastolisch 83,8 auf 79,5 mmHG sank (5). Ein Blutdruck-Rückgang bei Hypertonikern konnte auch in einer Analyse der TK beobachtet werden. Allerdings fiel auf, dass der Anteil von Hypertonikern mit der Zeit stieg.

Zudem zeigten Auswertungen des ZI aus der KV Nordrhein, dass zwar bei Patienten mit hohem Blutdruck der Wert über die Jahre von $188 \mathrm{mmHg}$ auf $146 \mathrm{mmHg}$ gesenkt werden konnte; bei gut eingestellten Patienten stieg er jedoch von $126 \mathrm{mmHg}$ auf $131 \mathrm{mmHg}$ an (6).

Der HbA1c lag bei Eintritt von AOK-Patienten ins DMP bereits bei einem recht günstigen Wert von 7; im sechsten Halbjahr der Auswertung betrug er 7,06 (5). TK-Versicherte starteten sogar mit noch günstigeren Werten in die DMP. Doch auch hier gab es keine anhaltende weitere Verbesserung in der Folgezeit.

In Auswertungen des ZI wird deutlich, dass die Gruppe mit einem hohen HbA1c-Wert sehr klein ist. Bei diesen Patienten mit einem Wert um 11,1 sank er von 2003 bis 2006 auf 8,5. Bei Patienten mit einem anfangs günstigen Wert von 5,9 Prozent stieg er jedoch auf 6,5 Prozent (6). 
Der BMI befand sich bei den AOK-Patienten im Mittel bei $30 \mathrm{~kg} / \mathrm{m} 2$ und blieb während des DMP auch auf diesem Niveau. Diese enttäuschende Beobachtung wird auch durch eine aktuelle Analyse für die Versicherten der TK bestätigt, die ebenfalls keine nennenswerten Effekte aufzeigen konnte.

Als Erklärung für diese Beobachtungen wird gelegentlich das über den DMP-Zeitraum zunehmende Alter der Versicherten und die natürliche Steigerung diverser Messparameter herangezogen. Damit bewegen wir uns jedoch wieder auf dem Gebiet der Hypothesen, die durch Evaluationen eigentlich bewiesen oder abgelehnt werden sollten.

Dies ist mit den meisten bisherigen Untersuchungen nicht möglich. Zum einen stimmen oftmals Ziele und Fragestellungen nur teilweise mit den Zielen der DMPVereinbarungen überein. So werden Kosten oder Vermeidung stationärer Behandlungen nicht betrachtet. Zum anderen wird in den meisten Studien keine Kontrollgruppe geführ. Eine Kausalität zwischen gemessenen Effekten und den Auswirkungen des Programms kann somit nicht belegt werden. Es können keine Aussagen getroffen werden, ob Veränderungen in den Ergebnissen ein Effekt des Krankheitsverlaufs, der politischen oder der wirtschaftlichen Entwicklung sind. Alle Interpretationsversuche bleiben Spekulation, was bei einem kostenaufwändigen Großversuch wie den DMP unverzeihlich ist.

Eine der wenigen Studien, die immerhin mit einer Kontrollkohorte Vergleiche anstellen, stammt vom Institut für Gesundheitsökonomie und Management im Gesundheitswesen. Die Autoren konnten in einer Evaluation eines DMP zur koronaren Herzkrankheit zeigen, dass die eingeschriebenen Patienten im Vergleich zur Standardversorgung häufiger ärztliche Beratung zu Ernährung und Bewegung erhielten. Unterschiede zeigten sich auch in einzelnen Kategorien der Lebensqualität (7).

Vergleichend war auch die ELSID-Studie, in der knapp 1400 Patienten befragt wurden, von denen zwei Drittel in DMP eingeschrieben waren. Sie kam zu der Erkenntnis, dass DMP-Patienten mit Ablauf und Organisation der Behandlung zufriedener sind als Patienten in der Regelversorgung (86 vs. 79 Prozent) (8).

Weller stieg in einer explorativen Studie noch weiter in psychologische Effekte ein und fand positive Auswirkungen der DMP-Teilnahme auf Aspekte wie Beziehung/Eingebundensein, Wissen/Bewusstsein, Lebensfreude/Optimismus etc. (9).

In keiner der Studien wurden jedoch die Zielparameter der DMP adäquat analysiert, obwohl verschiedene Autoren in der Vergangenheit Vorschläge für die Gestaltung sinnvoller Studiendesigns vorgelegt haben (10), (11).

Sicherlich ist es problematisch, eine Kontroll- oder Vergleichsgruppe zu rekrutieren, wenn eine Vielzahl der in Frage kommenden Patienten bereits in DMP eingeschlossen ist. Weitere Probleme bestehen darin, dass die Beobachtungszeiträume derzeit noch zu kurz sind, um patientenrelevante Outcomes wie das Auftreten von Schlagan- fällen, mikro- und makrovaskulären Komplikationen etc. analysieren zu können. Die teilnehmenden Praxen behandeln zudem überdurchschnittlich viele Diabetiker. Diese Probleme sind mit Studien, die in der Pilotphase eines DMP starten und nicht als Vollerhebungen angelegt sind, jedoch lösbar. Valide Designs sind auch relevant, wenn die Kosteneffektivität der Programm analysiert werden soll. Die Leistungsausgaben für DMP-Versicherte liegen deutlich über denen für Nicht-DMPPatienten, obwohl die eingeschriebene Population überproportional viele jüngere und gesündere Diabetiker erfasst (2). Diese wiederum gehören nicht zur Zielgruppe der Hochrisikopatienten, bei denen Effekte erzielt werden könnten.

Die Mehrausgaben für die Chroniker-Programme summierten sich zwischen 2003 und 2007 auf über drei Milliarden Euro. Allein 250 Millionen Euro fließen pro Jahr extrabudgetär in die ambulante ärztliche Versorgung.

Es werden damit große Teile der für die Regelversorgung der Bevölkerung zur Verfügung stehenden Finanzmittel gebunden. Je mehr Patienten in DMP eingeschrieben sind, desto geringer ist der Betrag, der einer Kasse für einen nicht eingeschriebenen Patienten mit derselben Erkrankung zur Verfügung steht. Es ist daher von größter Relevanz zu hinterfragen, ob dieses Geld gut investiert ist.

Bisher existiert jedoch keine Studie, in welcher die Kosteneffektivität der Programme evaluiert wird. Hierzu sind Langzeitbetrachtungen erforderlich, da erst durch die Vermeidung von schwerwiegenden Ereignissen wie Amputationen, Erblindung oder dialysepflichtigem Nierenversagen in der weiteren Zukunft eventuell ein Teil der investierten Kosten kompensiert werden kann.

Häussler berechnete, dass sich bei Annahme einer 25\%igen Zielwert-Einhaltung nach einer mehrjährigen Anlaufphase jährliche Einsparungen von bis zu 120 Millionen Euro pro Jahr ergeben, sofern Patienten über diese Zeit im DMP gehalten werden können. Dem gegenüber stehen jedoch Ausgaben in Höhe von 280 Millionen Euro pro Jahr (4).

Aber auch die Hoffnung, zumindest im Bereich der Arzneimittel Einsparungen zu erzielen, wurde nicht erfüllt. Die Ausgaben für die ambulante ärztliche Behandlung und Arzneimittel stiegen nach einer TK-Analyse vielmehr an. Einsparungen fanden in keinem Fall statt. Eine deutliche Reduktion von kostenaufwändigen Endpunkten war in der Kürze der Beobachtungszeit und mangels geeignetem Untersuchungsdesign bisher in keiner Studie aufzuzeigen.

Im Fazit wurde durch die Verknüpfung mit dem RSA somit nur die Einschreibung in die Programme und die Dokumentation, nicht jedoch eine bessere und kosteneffektive Versorgung von chronisch Kranken gefördert (12).

Wie passen die alten DMP in die neue Landschaft eines Morbi-RSA? Der Morbi-RSA soll Risikoselektion verringern und die Wirtschaftlichkeit der Leistungserbringung 
fördern. Die Kostenentwicklung soll begrenzt, die Bürokratie eingedämmt, Transparenz hergestellt, die Versorgungsqualität verbessert und Prävention gefördert werden. Zudem soll er Manipulationen verhindern und eine faire ambulante ärztliche Vergütung gewährleisten. Die meisten dieser Ziele werden durch die bisherigen DMP nicht unterstützt.

Daher war es eine kluge Entscheidung, mit Einführung des Morbi-RSA die Sonderstellung der DMP und die Verwebung von DMP und RSA zu beenden. Lediglich eine Programmkostenpauschale bleibt erhalten.

In Folge wird der Wettbewerb der Krankenkassen um möglichst viele Einschreibungen entfallen. Die Auswahl von Patienten müsste sich in Zukunft daran orientieren, wer von den DMP tatsächlich profitiert. Grundsätzlich haben nur DMP eine Überlebenschance, die kosteneffektiv sind. Welche das sind, kann mangels valider Studiendaten jedoch kaum ermittelt werden. Nach Wasem werden Programme, die zu Mehrkosten führen ohne dass ein Mehrnutzen erwartbar ist, nicht überleben. Und das sind prima vista praktisch alle.

Reduzierte Rekrutierungsaktivitäten der Krankenkassen, Dokumentationsmüdigkeit der Ärzte, unzureichende Evidenzbasierung der DMP, fragliche Verbesserung der Versorgungsqualität und primäre Kostensteigerungen ohne weiteren Abgleich der Mehrkosten über Ausgleichszahlungen werden das Ende von DMP der alten Machart einläuten - möglicherweise jedoch auch Impulse für neuartige Konzepte setzen.

\section{Ausblick}

Bevor jedoch weitere DMP in der Fläche gestartet werden, müssen diese in regional begrenzten Pilotvorhaben mit einem qualitativ hochwertigen Design auf ihren Nutzen und ihre Kosteneffektivität hin untersucht werden. Sie müssen hohen Qualitätsanforderungen entsprechen und sollten stärker die Verhaltensänderung des Patienten in den Mittelpunkt stellen. Verhaltensmodifizierende Betreuungsprogramme müssen ein zentraler Bestandteil der DMP sein, um nachhaltige Veränderungen des oft ungesunden Lebensstils der Patienten zu erzielen.

Es sollten nur Patienten behandelt werden, bei denen ein Potenzial für Behandlungserfolge besteht. Zu überdenken ist auch ein Vorschlag von Neubauer, der Treuerabatte für Patienten vorgeschlagen hat, je länger sie erfolgreich an der Änderung ihres Lebensstils arbeiten.

Innerhalb der DMP müssen verstärkt Patientenschulungen stattfinden und auch Ärzte müssen durch Fortbildungen an die Inhalte der DMP herangeführt werden. Die Programme sollten indikationsübergreifend ausge- richtet sein, da insbesondere bei multimorbiden Patienten oder solchen mit speziellen Risikokonstellationen Verbesserungspotentiale zu erwarten sind. Dazu sollten DMP in IV-Verträge integriert werden. Um die Versichertenbetreuung optimal zu unterstützen, muss jedoch eine versichertenbezogene Zusammenführung der erhobenen Daten über mehrere Leistungsbereiche hinweg bei der Krankenkasse zulässig werden.

All diese Maßnahmen werden jedoch auch in Zukunft primär zu Mehraufwendungen bei den Krankenkassen führen. Unterstützt der Gesetzgeber jedoch diesen Ansatz, der eine Chance auf erfolgreiche Umsetzung und nachhaltige Effekte hat, so sollte er die Programmkostenpauschale um Fixzuschläge für Versicherte ergänzen, wenn besondere Qualitätsanforderungen erfüllt werden.

\section{Literaturliste}

(1) Knieps F. DMP - Ein Erfolgsmodell. Eine Zwischenbilanz aus Sicht der AOK. AOK im Dialog 2007 Jun 26;21:19-25.

(2) Bullmann C, Straub C. DMP zwischen Anspruch und Wirklichkeit. Eigentlich sollte alles besser werden. Z Arztl Fortbild Qualitatssich 2006;100(1):32-5.

(3) Straub C. Disease-Management-Programme - Divergenzen zwischen Versorgungssteuerung und Risikostrukturausgleich. In: Klusen N, Straub C, Meusch A, editors. Steuerungswirkungen des Risikostrukturausgleichs.Baden-Baden: Nomos Verlagsgesellschaft; 2004. p. $140-9$.

(4) Häussler B, Berger U. Bedingungen für effektive Disease-Management-Programme. Analyse, Bewertung und Lösungsansätze für Qualität und Finanzierung. Baden-Baden: Nomos Verlagsgesellschaft; 2004.

(5) Eichenlaub A. Die gesetzliche Evaluation der Disease-ManagementProgramme. Ergebnisse der ersten AOK-Berichte zu den DMP Diabetes und Brustkrebs. AOK im Dialog 2007 Jun 26;21:33-41.

(6) Brenner G. Qualitätssicherung DMP. Das Beispiel der Qualitätsberichte zu den den nordrheinischen DMP. AOK im Dialog 2007 Jun 26; 49-57.

(7) Gapp O, Holle R, Meisinger C. Evaluation der Einschreibeselektion und gesundheitsökonomischer Aspekte bei Disease-ManagementProgrammen. Kongress Medizin und Gesellschaft 2007, Abstractband. 2007.

(8) Szecsenyi J. Besser als die Regelversorgung? Die ELSID-Studie zum Vergleich von optimal umgesetzten DMP, Routine DMP und Behandlung ohne DMP. AOK im Dialog 2008 Jun 26;21:59-67.

(9) Weller D. Explorative Studie zu positivem Teilnahmeerleben am DMP Diabetes mellitus Typ 2 in Westfalen-Lippe. Z Arztl Fortbild Qualitatssich 2005;99(3):201-7.

(10) Beyer M, Gensichen J, Szecsenyi J, Wensing M, Gerlach FM. Wirksamkeit von Disease-Management-Programmen in Deutschland - Probleme der medizinischen Evaluationsforschung anhand eines Studienprotokolls. Z ärztl Fortbild Qual Gesundh wes (ZaeFQ) 2006;100:355-63.

(11) Sawicki PT, Kraus HL, Heise T, Kaiser T, Gerlach FM, Gensichen J. Kontrollierte Evaluation der Effekte von Disease-Management-Programmen für Patienten mit Diabetes mellitus Typ 2 und Patienten mit koronarer Herzkrankheit. Frankfurt a.M.; 2006 Jul.

(12) Sachverständiger will DMP vom Kassenausgleich loseisen. Ärzte Zeitung 2007 Nov 12. 